\title{
Manual versus automatic identification of black-capped chickadee (Poecile atricapillus) vocalizations
}

\author{
Vala Ingolfsson ${ }^{1}$, William D. Service ${ }^{1}$, Carolina Montenegro ${ }^{1}$, Christopher B. Sturdy ${ }^{1}$ \\ ${ }^{1}$ Department of Psychology, Neuroscience and Mental Health Institute, University of Alberta
}

\begin{abstract}
One time-consuming aspect of bioacoustic research is identifying vocalizations from long audio recordings. SongScope (version 4.1.5. Wildlife Acoustics, Inc.) is a computer program capable of developing acoustic recognizers that can identify wildlife vocalizations. The goal of the current study was to compare the effectiveness of manual identification of black-capped chickadee vocalizations to identification by SongScope recognizers. A recognizer was developed for each main chickadee vocalization by providing previously annotated audio of chickadees. Six chickadees (three male, three female) were recorded in one-hour intervals with and without anthropogenic (i.e., man-made) noise to provide a variety of samples to test the recognizer. These recordings were analyzed via the recognizer and two human coders, with an additional third coder reviewing a random subset of recordings for reliability. Strong agreement was found between the human coders, $k=0.76, p<0.00$. Agreement between human coders and the recognizer was moderate for fee songs, $K=0.46, p<0.00$, and strong for fee-bee songs, $\mathrm{k}=0.77, \mathrm{p}<0.00$, as well as for chick-a-dee calls, $\mathrm{k}=0.82, \mathrm{p}<0.00$. Results showed that male chickadees produced more tseet calls in silence and females produced more gargle calls during noise. No differences were found in vocalizations based on time of day. Our observations also suggest that the chick-a-dee recognizer was capable of identifying gargle and tseet calls along with the intended chick-a-dee calls. Overall, SongScope was effective at identifying fee-bee songs and chick-a-dee calls, but not as effective for identifying fee songs. These recognizers can allow for faster acoustic analyses (by approximately four times) and be continuously improved for greater accuracy.
\end{abstract}

Key words:

bird calls, SongScope, black-capped chickadee, birds, vocalizations

Cite as: Ingolfsson V., Service W.D., Montenegro C., and Sturdy C.B. 2019. Manual versus automatic identification of black-capped chickadee (Poecile atricapillus) vocalizations. Alberta Academic Review, Vol 2 (2) 41-42, WISEST Special Issue (not peer-reviewed), DOI 10.29173/ aar48. 
Ingolfsson et al., 2019

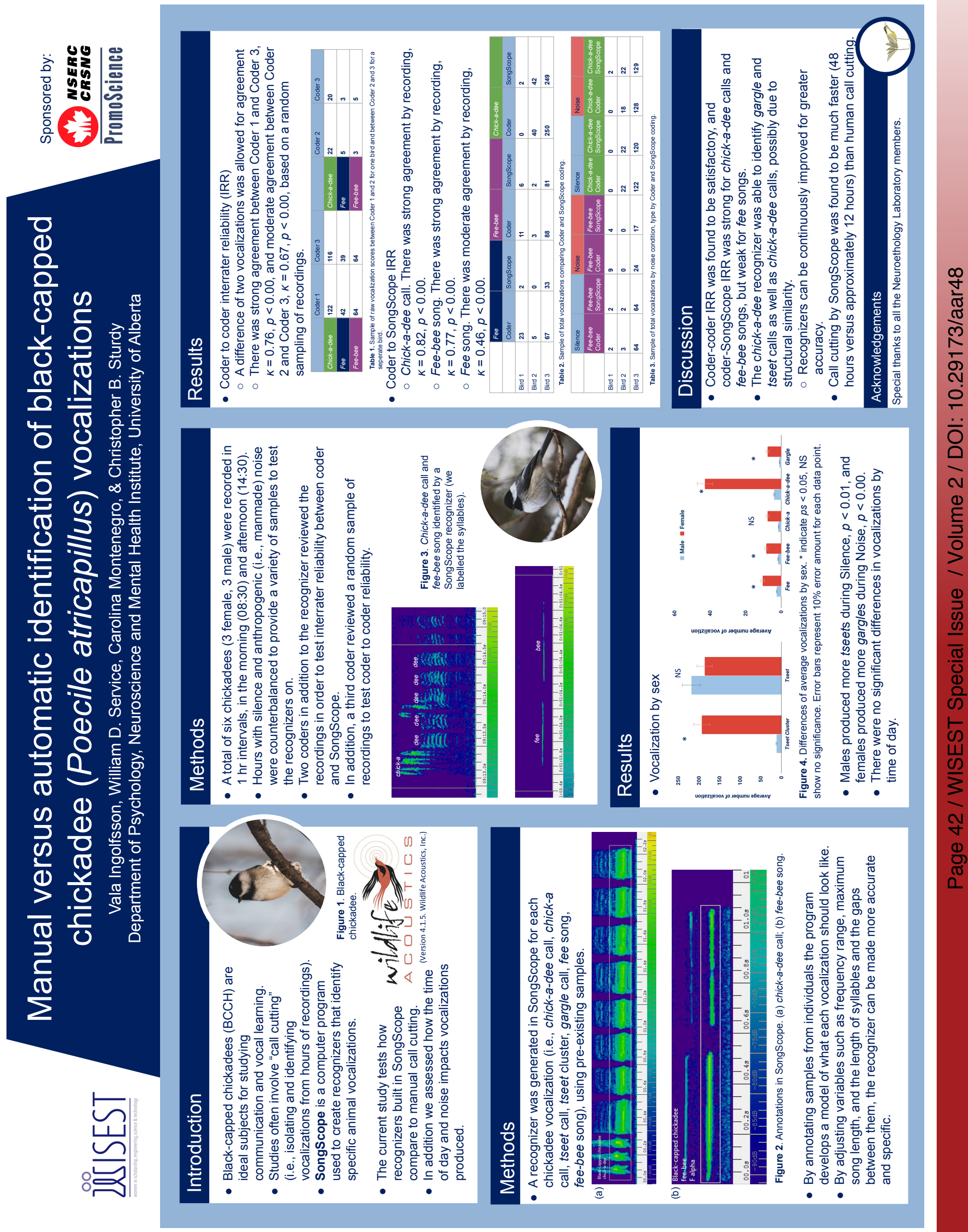

